INT. J. SYSTEMS SCI., 1988, vOL. 19, NO. $6,1011-1020$

\title{
Output-maximal control policies for cascaded production-inventory systems with control and state constraints
}

\section{J. WARSCHAT $\dagger$ and H. J. WUNDERLICH $\ddagger$}

Optimal control policies are derived for cascaded production-inventory systems. As objectives, output maximization and the minimum time to produce a fixed output are considered. An example consisting of three subsystems is detailed to illustrate the proposed theory.

\section{Introduction}

This paper is concerned with the optimal planning of production over a fixed time horizon $[0, T]$ for cascaded production-inventory systems as described by Bradshaw and Erol (1980). A subsystem $j$ consists of a plant and of an inventory. The plant has an actual production rate $p_{j}(t)$ which follows the desired production rate $p_{d j}(t)$ with a first-order time delay with time constant $1 / \alpha_{j}$. The actual inventory level $i(t)$ is the balance of the actual production rate and the shipping rate $s_{j}(t)$. Therefore, the subsystem $j$ is governed by the differential equations:

$$
\begin{aligned}
\dot{i}_{j}(t) & =p_{j}(t)-s_{j}(t) \\
\dot{p}_{j}(t) & =\alpha_{j}\left(p_{d j}(t)-p_{j}(t)\right)
\end{aligned}
$$

In the following we consider a cascade of $m$ subsystems (1) and (2) which are connected by

$$
s_{j+1}(t)=p_{d j}(t), \quad j=1, \ldots, m
$$

i.e. the output of a subsystem is equal to the input of the subsequent subsystem as shown in Fig. 1.

Regarding the desired production rates $p_{d j}(t)$ as control variables $u_{j}(t)$ and the actual production rates $p_{j}(t)$ and the inventory levels $i_{j}(t)$, as state variables $x_{2 j}(t)$ and $x_{2 j-1}(t), j=1, \ldots, m$, we get the state equation

$$
\dot{x}(t)=A x(t)+B u(t)
$$

where

$$
x^{T}(t)=\left[\begin{array}{lll}
x_{1} & \ldots & x_{2 m}
\end{array}\right], \quad u^{T}(t)=\left[\begin{array}{lll}
u_{1} & \ldots & u_{m}
\end{array}\right]
$$

Received 4 December 1986.

‡ Fraunhofer Institute for Industrial Engineering, Nobelstrasse 12C, D-7000 Stuttgart 80, West Germany.

$\ddagger$ Institut für Informatik IV, Universität Karlsruhe, Zirkel 2, D-7500 Karlsruhe 1, West Germany. 
and

$$
A=\left[\begin{array}{ccccccc}
0 & 1 & 0 & 0 & \ldots & 0 & 0 \\
0 & -\alpha_{1} & 0 & 0 & \ldots & 0 & 0 \\
0 & 0 & 0 & 1 & \ldots & 0 & 0 \\
0 & 0 & 0 & -\alpha_{2} & \ldots & 0 & 0 \\
\vdots & \vdots & \vdots & \vdots & & \vdots & \vdots \\
0 & 0 & 0 & 0 & \ldots & 0 & 1 \\
0 & 0 & 0 & 0 & \ldots & 0 & -\alpha_{m}
\end{array}\right], B=\left[\begin{array}{cccccc}
0 & 0 & 0 & \ldots & 0 & 0 \\
\alpha_{1} & 0 & 0 & \ldots & 0 & 0 \\
-1 & 0 & 0 & \ldots & 0 & 0 \\
0 & \alpha_{2} & 0 & \ldots & 0 & 0 \\
\vdots & \vdots & \vdots & & \vdots & \vdots \\
0 & 0 & 0 & \ldots & -1 & 0 \\
0 & 0 & 0 & \ldots & 0 & \alpha_{m}
\end{array}\right]
$$

with the initial and end conditions

$$
\left.\begin{array}{r}
x(0)=x_{0} \\
x(T)=x_{T}
\end{array}\right\}
$$

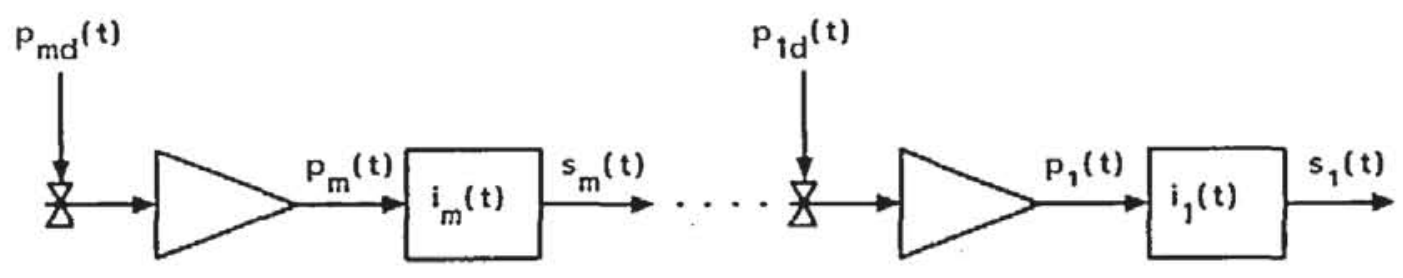

Figure 1.

In most cases the production rates have to remain within certain limits where we can set

$$
\zeta_{j} \leqq u_{j}(t) \leqq \xi_{j}, \quad \forall t \in[0, T]
$$

without loss of generality we assume $\zeta_{j}=-1, \xi_{j}=+1$, and often the state variables are restricted in a similar manner, for example the inventories may not violate a safety stock

$$
x_{2 j-1}(t) \geqq \beta_{j}, \quad \forall t \in[0, T]
$$

or the actual production rates cannot exceed a maximum rate

$$
x_{2 j}(t) \leqq \beta_{j}, \quad \forall t \in[0, T]
$$

The objective is the output maximization of the system, i.e. the maximization of the inventory at time $T$ of the last subsystem, which implies $s_{1}(t) \equiv 0$ :

$$
J(u)=-x_{1}(T) \rightarrow \min
$$

In a recent paper (Warschat and Wunderlich 1984) control policies were derived concerning the time-optimal state transition of system (4) with totally prescribed initial and end conditions (5). These policies were attained by application of a theory for state-constrained systems developed by Jacobsen et al. (1971), Hamilton (1972) and Maurer (1977). In this paper it is shown that output-maximal and time-minimal policies for a fixed output, where only the end conditions of the inventories are prescribed, can be found in a similar way. For demonstration, the problem is stated in a more general form; see Jacobsen et al. (1971), Hamilton (1972) and Maurer (1977). 


\section{Necessary conditions}

Determine a piecewise continuous control $u(t), t \in[0, T]$, which minimizes the functional

$$
J(u):=F(x(T))
$$

subject to

$$
\begin{aligned}
\dot{x}(t) & =f(x(t), u(t)):=f_{1}(x(t))+f_{2}(x(t)) u(t) \\
x(0) & =x_{0}, \quad Q(x(T))=0 \\
u(t) & \in U:=\left\{u \in \mathbb{R}^{m} \mid-1 \leqq u_{j} \leqq 1, j=1, \ldots, m\right\} \\
S_{i}(x(t)) & \leqq 0, \quad i=1, \ldots, h
\end{aligned}
$$

with the state vector $x(t) \in \mathbb{R}^{n}$, the control vector $u(t) \in \mathbb{R}^{m}$, the functions $F: \mathbb{R}^{n} \rightarrow \mathbb{R}$, $f_{1}: \mathbb{R}^{n} \rightarrow \mathbb{R}^{n}, f_{2}: \mathbb{R}^{n} \rightarrow \mathbb{R}^{n \times m}, Q: \mathbb{R}^{n} \rightarrow \mathbb{R}^{k}, k<n, S: \mathbb{R}^{n} \rightarrow \mathbb{R}^{h}, h \leqq m$, which are assumed to be continuously differentiable for a sufficient number of times.

\section{Definition}

The interval $I:=\left[t_{1}, t_{2}\right] \subset[0, T]$ is called the boundary arc and $t_{1}$ and $t_{2}$ are called the entry and the exit time, respectively, of the trajectory $x(t)$ concerning $S_{i}$, if there exists a $\varepsilon>0$ with $S_{i}(x(t)) \neq 0$ for $t_{1}-\varepsilon \leqq t<t_{1}$ and for $t_{2}<t \leqq t_{2}+\varepsilon$ and $S_{i}(x(t))=0$ for $t \in I$.

Now we use Maurer's form of the minimum principle.

$(M P)$. For each optimal solution $x(t), u(t)$ of the problem (10)-(14) there exists an adjoined vector $\lambda(t) \in \mathbb{R}^{n}$ and a measurable function $\eta:[0, T] \rightarrow \mathbb{R}^{h}$ with $\eta^{T}(t) S(x(t)) \equiv 0$, $t \in[0, T]$, fulfilling (15)-(19):

$$
\begin{aligned}
\lambda^{T} & =-\lambda^{T} f_{1 x}(x)-\lambda^{T} f_{2 x}(x) u-\eta^{T} S_{x}(x) \\
\lambda^{T}(T) & =F_{x}(x(T))+\sigma^{T} Q_{x}(x(T)), \quad \sigma \in \mathbb{R}^{k}
\end{aligned}
$$

Additionally, we assume here the Slater condition (see Girsanov 1972).

$$
\lambda^{T}\left(t^{+}\right)=\lambda^{T}\left(t^{-}\right)-v^{T}(t) S_{x}(x(t)), \quad v_{i}(t) \geqq 0, \quad i=1, \ldots, h
$$

where

$$
v(t):=\eta\left(t^{+}\right)-\eta\left(t^{-}\right)
$$

With the hamiltonian

$$
\begin{aligned}
H(x(t), u(t), \lambda(t), \eta(t)):= & \lambda^{T}(t) f_{1}(x(t))+\lambda^{T}(t) f_{2}(x(t)) u(t) \\
& +\eta^{T}(t) S(x(t))
\end{aligned}
$$

we get the minimum principle

$$
H(x(t), u(t), \lambda(t), \eta(t))=\min _{u \in U} H(x(t), u, \lambda(t), \eta(t))
$$

Because of the form of the hamiltonian which contains the control vector linearly, we define a switching function in order to determine $u(t)$ :

$$
\phi^{T}(t):=\lambda^{T}(t) f_{2}(x(t))
$$


For each component $\phi_{j}^{T}(t) \neq 0$, the minimum principle (19) yields the bang-bang control:

$$
u_{j}(t)=\left\{\begin{aligned}
-1 & \text { if } \phi_{j}(t)>0 \\
1 & \text { if } \phi_{j}(t)<0
\end{aligned}\right.
$$

\section{Output maximization}

\subsection{Unconstrained trajectories}

For ease of notation, we transfer (4) without loss of generality into

$$
\dot{x}(t)=T A x(t)+T B u(t), \quad t \in[0,1]
$$

To avoid any ambiguity we set the initial conditions to

$$
x(0)=0
$$

For other values the modifications of the following arguments are obvious. As final conditions, we assume fixed inventories and free production rates, i.e.

$$
x_{2 j-1}(1)=a_{j}, \quad x_{2 j}(1) \text { free, } j=2, \ldots, m
$$

For determined $x_{2 j-1}(1)$ and $x_{2 j}(1)$, the theory developed by Warschat and Wunderlich (1984) holds. Clearly the $a_{j}$ have to be restricted to ensure a solution of the problem. Therefore, we define in the following the admissible end conditions by

$$
\left|\frac{a_{j}}{T} \pm 1\right|>\left|1-\frac{1-\exp \left(-T \alpha_{j}\right)}{T \alpha_{j}}\right| \quad(T \text { finite })
$$

which describe the open set of controllable output. For $a_{j}=0,(25)$ is always fulfilled.

The adjoined equation is

$$
\dot{\lambda}(t)=-T A^{T} \lambda(t)
$$

(MP) yields

$$
\left.\begin{array}{c}
\lambda_{1}(1)=-1=: b_{1} \\
\lambda_{2 j-1}(1)=b_{2 j-1}, \quad j=2, \ldots, m \\
\lambda_{2 j}(1)=0=: b_{2 j}, \quad j=1, \ldots, m
\end{array}\right\}
$$

Hence we get from (26) by integration

$$
\begin{aligned}
\lambda_{2 j-1}(t) & =b_{2 j-1}, \quad t \in[0,1] \\
\lambda_{2 j}(t) & =-\frac{b_{2 j-1}}{\alpha_{j} \exp \left(T \alpha_{j}\right)} \exp \left(T \alpha_{j} t\right)+\frac{b_{2 j-1}}{\alpha_{j}}, \quad t \in[0,1]
\end{aligned}
$$

From (20) we get, with respect to (22), for each component:

$$
\left.\begin{array}{l}
\phi_{j}=\alpha_{j} \lambda_{2 j}-\lambda_{2 j+1}, \quad j=1, \ldots, m-1 \\
\phi_{m}=\alpha_{m} \lambda_{2 m}
\end{array}\right\}
$$

Taking (29) and (30) into consideration, the switching functions may be constant or may have at most one switching point. Singular controls occur if $\phi_{j} \equiv 0$, which implies $b_{2 j-1}=0$ and $b_{2 j+1}=0$. Therefore $u_{1}$ must be a bang-bang control. 


\section{Lemma}

All $u_{j}, j=1, \ldots, m$ are bang-bang controls.

The proof runs by contradiction. Let $j \geqq 2$ be the smallest integer with a singular $u_{j}$. Consequently, $\phi_{j} \equiv 0$ holds and therefore we have $b_{2 j-1}=0, \phi_{j-1}=\alpha_{2 j-1} \lambda_{2 j-1}$ and $\phi_{j-1}(1)=0$. The function $\phi_{j-1}$ is monotonous. Therefore the bang-bang control $u_{j-1}$ has no switching point and $u_{j-1} \equiv 1$ or $u_{j-1} \equiv-1$ must hold. Integrating (22) with respect to (23) we get

$$
x_{2 j}(t)=T \alpha_{j} \exp \left(-T \alpha_{j} t\right) \int_{0}^{t} u_{j}(\tau) \exp \left(T \alpha_{j} \tau\right) d \tau
$$

and

$$
a_{j}=x_{2 j-1}(1)=T\left(\int_{0}^{1}\left(T \alpha_{j} \exp \left(-T \alpha_{j} t\right) \int_{0}^{t} u_{j}(\tau) \exp \left(T \alpha_{j} \tau\right) d \tau\right) d t-u_{j-1}\right)
$$

With Fubini's theorem, (33) can be transformed to

$$
\begin{aligned}
a_{j} / T+u_{j-1} & =\int_{0}^{1} u_{j}(\tau) \exp \left(T \alpha_{j} \tau\right) T \alpha_{j} \int_{\tau}^{1} \exp \left(-T \alpha_{j} t\right) d t d \tau \\
& =\int_{0}^{1} u_{j}(\tau) \exp \left(T \alpha_{j} \tau\right)\left(\exp \left(-T \alpha_{j} \tau\right)-\exp \left(-T \alpha_{j}\right)\right) d \tau \\
& =\int_{0}^{1} u_{j}(\tau)\left(1-\exp \left(-T \alpha_{j}\right) \exp \left(T \alpha_{j} \tau\right)\right) d \tau
\end{aligned}
$$

The function $\left(1-\exp \left(-T \alpha_{j}\right) \exp \left(T \alpha_{j} \tau\right)\right)$ is non-negative on $[0,1]$. Thus there exists a $\rho \in[-1,1]$ with

$$
\int_{0}^{1} u_{j}(\tau)\left(1-\exp \left(-T \alpha_{j}\right) \exp \left(T \alpha_{j} \tau\right)\right) d \tau=\rho \int_{0}^{1}\left(1-\exp \left(-T \alpha_{j}\right) \exp \left(T \alpha_{j} \tau\right)\right) d \tau
$$

Evaluating the integrals we obtain

$$
a_{j} / T+u_{j-1}=\rho\left(1-\frac{1-\exp \left(-T \alpha_{j}\right)}{T \alpha_{j}}\right)
$$

But $\left|u_{j-1}\right|=1$ yields

$$
\left|a_{j} / T \pm 1\right|=|\rho|\left|1-\frac{1-\exp \left(-T \alpha_{j}\right)}{T \alpha_{j}}\right| \leqq\left|1-\frac{1-\exp \left(-T \alpha_{j}\right)}{T \alpha_{j}}\right|
$$

This contradicts (25).

It only remains to determine the switching point $t_{j}$ for each $u_{j}$. Dealing with output maximization it will be obvious to start with positive controls. Otherwise, the modifications in the subsequent relations are straightforward. Let $j=2, \ldots, m$, then

$$
\begin{aligned}
a_{j} & =T \int_{0}^{1} u_{j}(\tau)\left(1-\exp \left(-T \alpha_{j}\right) \exp \left(T \alpha_{j} \tau\right)\right) d \tau-T \int_{0}^{1} u_{j-1}(\tau) d \tau \\
& =T\left(2 t_{j}-1-\frac{2 \exp \left(-T \alpha_{j}\right) \exp \left(T \alpha_{j} t_{j}\right)-1-\exp \left(-T \alpha_{j}\right)}{T \alpha_{j}}\right)-2 T t_{j-1}+T
\end{aligned}
$$


From $\phi_{m}(1)=\alpha_{2 m} \lambda_{2 m}(1)=0$ we get $t_{m}=1$. So we can determine the $t_{j}, j=m-1, \ldots, 1$, recursively from (34):

$t_{j}=\left(2 t_{j+1}-\frac{2 \exp \left(-T \alpha_{j+1}\right) \exp \left(T \alpha_{j+1} t_{j+1}\right)-1-\exp \left(-T \alpha_{j+1}\right)}{T \alpha_{j+1}}-\frac{a_{j+1}}{T}\right) / 2$

Then the optimal controls solving our problem become

$$
\bar{u}_{j}(t)=\left\{\begin{aligned}
1, & 0 \leqq t \leqq t_{j} \\
-1, & \text { otherwise }
\end{aligned}\right.
$$

Note: If the final condition for an inventory is free, i.e. one of the $a_{j}, j=1, \ldots, m$, for example $a_{k}$, is not defined, the respective control $u_{k}$ is singular and can be chosen as a suitable constant. Then it follows from the previous discussion that $u_{k-1} \equiv 1$.

\subsection{Constrained trajectories}

Now the trajectories have to satisfy additionally the state constraints (7) or (8) which leads, using (14), to

$$
S_{i}(x(t)):=\left\{\begin{array}{c}
x_{2 j}(t)-\beta_{2 j} \\
\beta_{2 j+1}-x_{2 j+1}(t)
\end{array}\right\} \leqq 0, \quad i=1, \ldots, h
$$

The first derivative of (37) is

$$
\dot{S}_{i}(x(t))=\dot{x}_{2 j}(t)=-\alpha_{j} x_{2 j}(t)+\alpha_{j} u_{j}(t)=0
$$

so we get the boundary control $u_{j}(t)=x_{2 j}(t)=\beta_{2 j}$. Analogously, we obtain the boundary control for (38); $u_{j}(t)=x_{2 j+2}(t)$.

For both constraints the first derivative of $S_{i}(x(t))$ contains $u(t)$ explicitly. Maurer (1977) has shown that in this case the adjoined vector is continuous at entry and exit points. Therefore, the basic considerations of the previous section remain valid, but in addition we have to determine the entry and the exit points concerning the boundary controls.

In the following we obtain the unknown control functions $u_{j}(t), j=m, \ldots, 1$ by a recursive computation of the corresponding switching points $t_{j}^{1}$ and $t_{j}^{2}$. Thereby we do not deal with cases where both $x_{2 j+1}$ and $x_{2 j}$ are restricted, because this would not change the arguments but would cause more effort in notation.

(1) $j=m$.

(a) If $x_{2 m}$ is not restricted, set $u_{m}(t)=1$.

(b) There is a boundary $x_{2 j}(t)-\beta_{2 j} \leqq 0$.

If we set $x_{2 j}\left(t_{m}^{1}\right)=\beta_{2 j}$ we get with (32)

$$
t_{m}^{1}=-\frac{\ln \left(1-\beta_{2 j}\right)}{T \alpha_{j}}
$$

Thus the control is

$$
u_{m}(t)= \begin{cases}1, & 0 \leqq t \leqq t_{m}^{1} \\ \beta_{2 j}, & t_{m}^{1}<t \leqq 1\end{cases}
$$

(2) $j=l-1, l=2, \ldots, m$. 
(a) For an unconstrained subsystem $j$, the switching point is determined by (34):

$$
\left.\begin{array}{c}
a_{1}=T \int_{0}^{1} u_{1}(\tau)\left(1-\exp \left(-T \alpha_{1}\right) \exp \left(T \alpha_{1} \tau\right)\right) d \tau-T \int_{0}^{1} u_{j}(\tau) d \tau \\
t_{j}^{1}=\left(\int_{0}^{1} u_{1}(\tau)\left(1-\exp \left(-T \alpha_{1}\right) \exp \left(T \alpha_{1} \tau\right)\right) d \tau-a_{1} / T+1\right) / 2
\end{array}\right\}
$$

The control is

$$
u_{j}(t)=\left\{\begin{array}{rr}
1, & 0 \leqq t \leqq t_{j}^{1} \\
-1, & t_{j}^{1}<t \leqq 1
\end{array}\right.
$$

(b) $x_{2 j}-\beta_{2 j} \leqq 0$.

We compute $t_{j}^{1}$ as in $(1(b))$, then we proceed as in $(2(a))$ :

$$
\left.\begin{array}{c}
a_{1}=T\left(\int_{0}^{1} u_{1}(\tau)\left(1-\exp \left(-T \alpha_{1}\right) \exp \left(T \alpha_{1} \tau\right)\right) d \tau-T\left(t_{j}^{1}+\beta_{2 j}\left(t_{j}^{2}-t_{j}^{1}\right)-\left(1-t_{j}^{2}\right)\right)\right. \\
t_{j}^{2}=\left(\int_{0}^{1} u_{1}(\tau)\left(1-\exp \left(-T \alpha_{1}\right) \exp \left(T \alpha_{1} \tau\right)\right) d \tau-a_{1} / T+t_{j}^{1}\left(\beta_{2 j}-1\right)+1\right) /\left(\beta_{2 j}+1\right)
\end{array}\right\}
$$

As control we get:

$$
u_{j}(t)=\left\{\begin{aligned}
1, & 0 \leqq t<t_{j}^{1} \\
\beta_{2 j}, & t_{j}^{1} \leqq t \leqq t_{j}^{2} \\
-1, & t_{j}^{2}<t \leqq 1
\end{aligned}\right.
$$

(c) $\beta_{2 j+1}-x_{2 j+1} \leqq 0$

The entry point is computed by integrating the trajectory until $t_{t}^{1}$ :

$$
\begin{aligned}
\beta_{2 j+1} & =T \int_{0}^{t_{j}^{j}}\left(\exp \left(-T \alpha_{1} t\right) T \alpha_{1} \int_{0}^{t} u_{1}(\tau) \exp \left(T \alpha_{1} \tau\right) d \tau-u_{j}(t)\right) d t \\
& =T \int_{0}^{t_{j}^{j}} u_{1}(\tau)\left(1-\exp \left(-T \alpha_{1} t_{j}^{1}\right) \exp \left(T \alpha_{1} \tau\right)\right) d \tau-T t_{j}^{1}
\end{aligned}
$$

The exit point $t_{j}^{2}$ is obtained by:

$$
a_{1}=x_{2 j+1}(1)=\beta_{2 j+1}+T \int_{t^{2}}^{1}\left(x_{2 l}(t)+1\right) d t
$$

The control becomes:

$$
u_{j}(t)=\left\{\begin{array}{cc}
1, & 0 \leqq t<t_{j}^{1} \\
x_{21}(t), & t_{j}^{1} \leqq t \leqq t_{j}^{2} \\
-1, & t_{j}^{2}<t \leqq 1
\end{array}\right.
$$

This completes the recursion. 

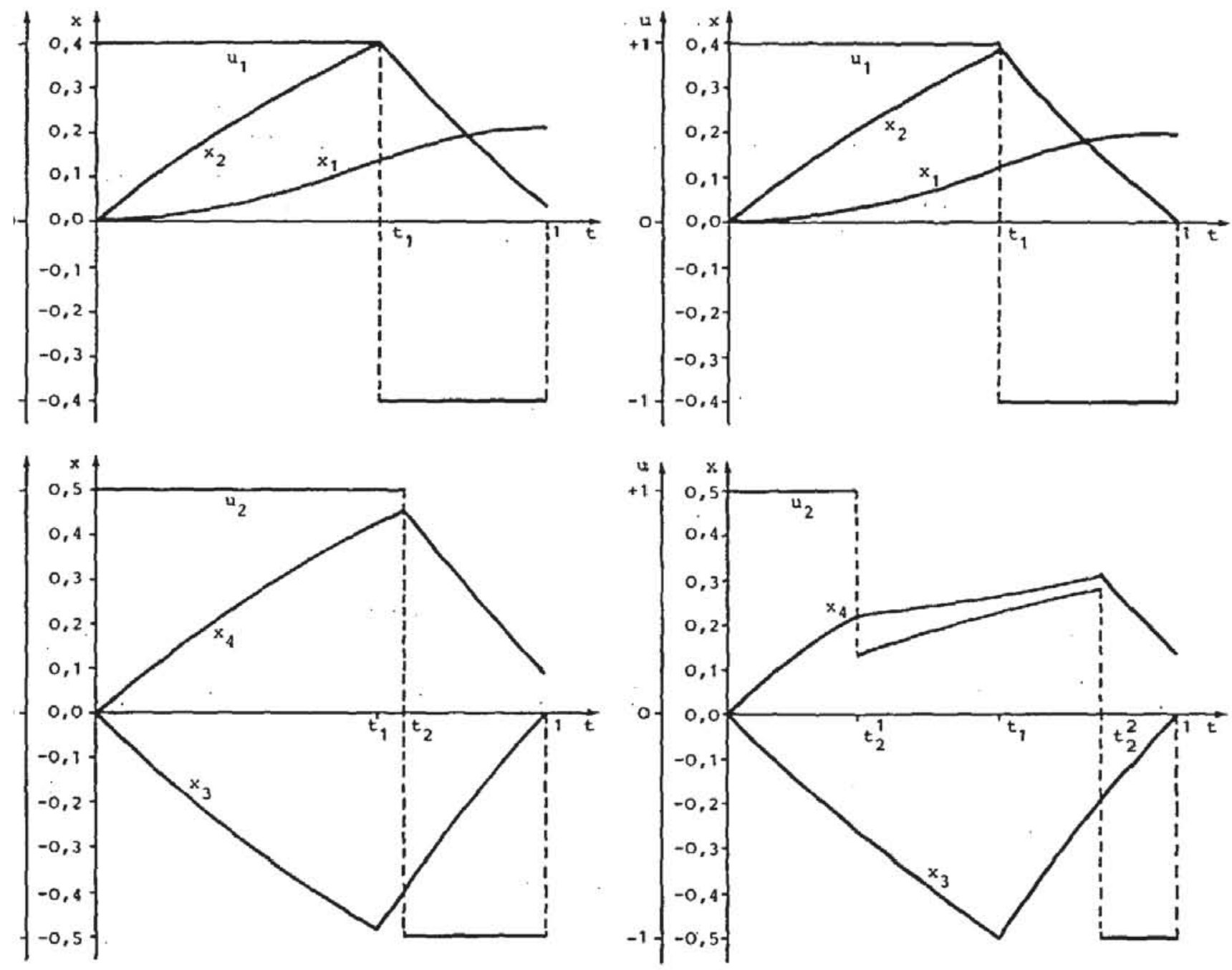

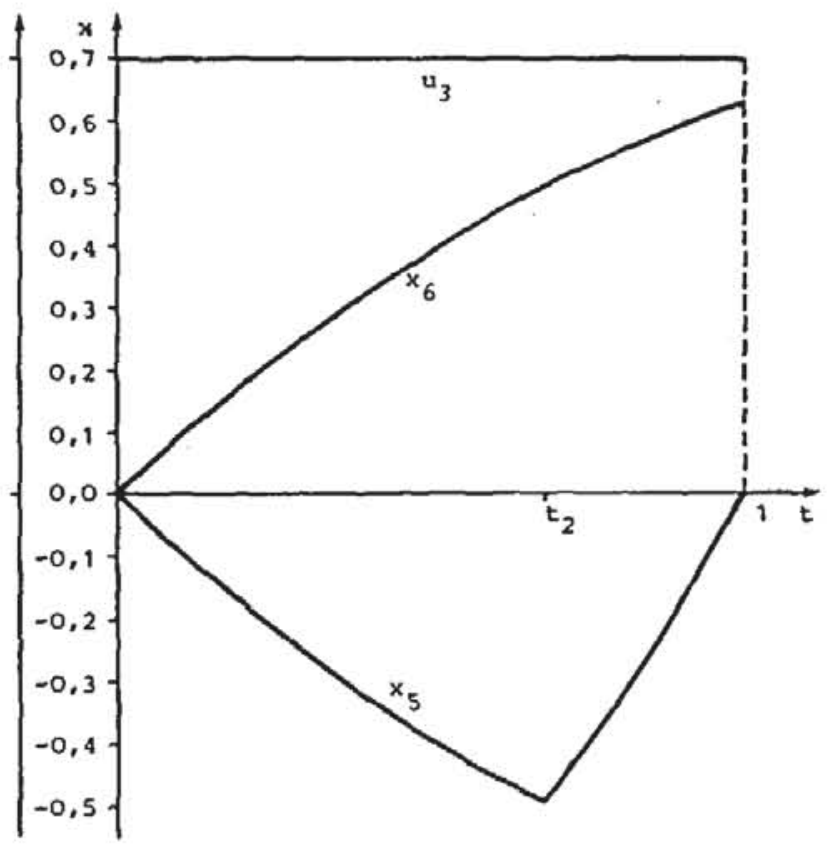

Constrained case.

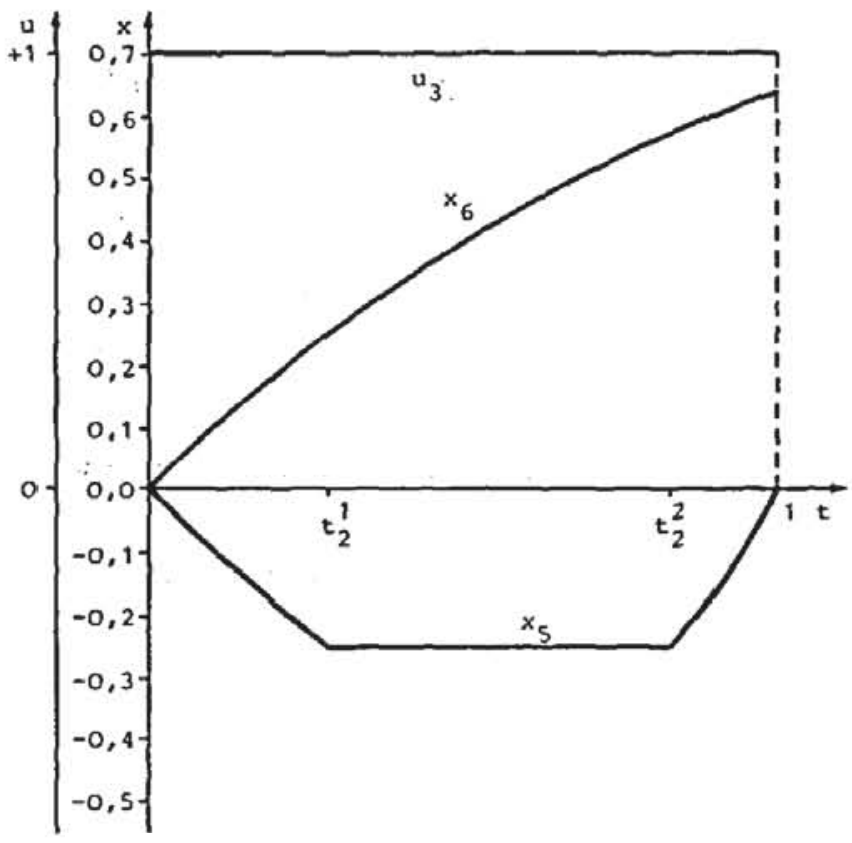

Unconstrained case.

Figure 2. 


\section{Time-minimization for fixed output}

In order to supply the representation of the previous sections we consider another objective function

$$
J(u)=T \rightarrow \min
$$

where the output of the last subsystem is fixed

$$
x_{1}(1)=a
$$

The other relations remain valid. In a sense this is the 'inverse' planning problem to (9).

For each $T>0$, let $a(T)$ denote the maximal output $x_{1}(1)=a(T)$ of the system (22). Then $T_{1}>T_{2}$ yields $a\left(T_{1}\right)>a\left(T_{2}\right)$. If there exists a $T>0$ with $a=a(T)$, then $T$ is the minimal time to produce $a$. For each $T>0$, let $u_{j}^{T}$ be determined as in (36) or in (40), (42), (44), (47), respectively. We define

$$
\Delta(T):=a-x_{1}(1)=a-T \int_{0}^{1} u_{1}^{T}(\tau)\left(1-\exp \left(-T \alpha_{1}\right) \exp \left(T \alpha_{1} \tau\right)\right) d \tau
$$

Now it suffices to look for a zero $\Delta(T)=0$ of the real function (50). So we get the minimal time $T$ and the corresponding optimal control $u_{j}^{T}$.

\section{Numerical example}

In order to illustrate the ideas of the previous sections we consider a productioninventory system consisting of three subsystems. The constants of the time delays are $\alpha_{1}=0.8, \alpha_{2}=0.9, \alpha_{3}=1 \cdot 0$. The optimal control policies and the respective state variables for the output maximization are shown in the left-hand side of Fig. 2. No constraint is active. The interval $T$ is chosen as 1 . For simplicity, all state variables start with 0 as initial value. Because of the impossibility of negative inventories, this means that one has to provide safety stocks of at least the maximal amount of $x_{2 j-1}(t), j=1,2,3$.

Subsystem three which lies on the input side of the system works over the whole interval with maximal $u_{1}(t)\left(t_{3}=1 \cdot 0\right)$. This is possible if we assume that there is always enough input available. Subsystems one and two are bang-bang controlled with switching times $t_{1}=0.6293$ and $t_{2}=0.6839$, respectively. The output of the system is $x_{1}(1)=0 \cdot 2119$. Now we impose a constraint on the inventory $x_{5}(t)$ (see case $(2(b))$ ) $S:=0.25-x_{5}(t) \leqq 0$. This can be interpreted as too small a safety stock of system three. This system works with maximal production rate $\left(t_{3}=1 \cdot 0\right)$ but, due to the constraint, the control policy $u_{2}(t)$ has to follow exactly the production rate $x_{6}(t)$ in the interval $\left[t_{2}^{1}, t_{2}^{2}\right]$, with $t_{2}^{1}=0.2877$ and $t_{2}^{2}=0.8439$, since the output of $x_{5}(t)$ can be at most equal to the input.

\section{Conclusion}

In this paper we consider cascaded production-inventory systems. We present optimal control policies for the problems of output maximization and of time-optimal production of a fixed output where the control variables are bounded. It is shown that all subsystems are bang-bang controlled. Then we regard the state-constrained case where we distinguish inventory and production rate constraints. For both problems we get the optimal control policies and the respective optimal trajectories with the entry and exit times at the bounded arcs. 


\section{ACKNOWLEDGMENT}

This research was supported by Stiftung Volkswagenwerk.

\section{REFERENCES}

Bradshaw, A., and Erol, Y., 1980, Int. J. Systems Sci., 11, 947.

Girsanov, I. V., 1972, Lectures on Mathematical Theory of Extremum Problems (Berlin: Springer-Verlag).

Hamilton, W. E., 1972, I.E.E.E. Trans. autom. Control, 17, 338.

Jacobsen, D. H., Lele, M. M., and Speyer, J. L., 1971, J. math. Analysis Applic., 35, 255.

MAURER, H., 1977, SIAM J. Control, 15, 345.

WARSChat, J., and WUNDERLich, H. J., 1984, Int. J. Systems Sci., 15, 513. 